Original Article

\title{
Finite-Difference Scheme for Initial Boundary Value Problems in Financial Mathematics
}

\author{
Le Minh Hieu ${ }^{1}$, Truong Thi Hieu Hanh ${ }^{1}$, Dang Ngoc Hoang Thanh ${ }^{2, *}$ \\ ${ }^{1}$ University of Economics, The University of Danang, 71 Ngu Hanh Son, Da Nang, Vietnam \\ ${ }^{2}$ Hue College of Industry, 70 Nguyen Hue, Hue, Vietnam
}

Received 02 August 2019

Accepted 11 September 2019

\begin{abstract}
This study develops unconditionally monotone finite-difference scheme of second-order of local approximation on uniform grids for the initial boundary problem value for the Gamma equation through the establishment of two-side estimates for the scheme's solution. The study considers the initial boundary value problem for the so called Gamma equation, which can be derived by transforming the nonlinear Black-Scholes equation for option price into a quasilinear parabolic equation for the second derivative of the option price. By means of regularization principle, the previous study results were generalized for construction of unconditionally monotone finite-difference scheme (the maximum principle was satisfied without constraints on relations between the coefficients and grid parameters) of second order of approximation on uniform grids for this equation. With the help of difference maximum principle, the two-side estimates for difference solution were obtained at the arbitrary non-sign-constant input data of the problem. A priori estimate in the maximum norm $\mathrm{C}$ was proved. Interestingly, the proven two-side estimates for difference solution were fully consistent with differential problem, and the maximal and minimal values of the difference solution did not depend on the diffusion and convection coefficients. Finally, relevant computational experiments were given to confirm the above-named theoretical findings.
\end{abstract}

Keywords: Gamma equation, maximum principle, two-side estimates, monotone finite-difference scheme, quasi-linear parabolic equation, scientific computing.

\section{Introduction}

Over the last decades, not only financial engineers but also mathematicians have paid special attention to the valuation of derivative financial instruments. Indeed, since being introduced by Fischer

\footnotetext{
${ }^{*}$ Corresponding author.

Email address: hieulm@due.edu.vn
}

https//doi.org/ 10.25073/2588-1124/vnumap.4364 
Black and Myron Scholes in 1973, the Black-Scholes model based on partial differential equation has been widely employed in modern mathematical finance and become a common-sense approach for pricing options as well as many other financial securities [1]. This mathematical model was derived from the principle that yielding profits from making portfolios of both short and long positions in options as well as their underlying stocks should not be possible, if option prices are rightly priced in the market [2]. These scholars indicated that a European option's value on a stock, whose price or the log return of underlying price is supposed to follow a geometric Brownian motion with constant volatility and drift, is determined by a second-order parabolic equation concerning time and stock price. Nevertheless, the assumptions of Black-Scholes equation based on perfectly liquid market are so idealistic in comparison with the high illiquidity recently.

A variety of numerical methods were used in previous papers for studying properties of typical nonlinear Black-Scholes equations, see, for instance [3-5] and references there in.

Not only in mathematical physics, but also in economics, there is a need to solve partial differential equations containing lower derivatives. For example, in financial mathematics, it is of interest to study the Gamma equation obtained by transforming the nonlinear Black-Scholes equation into a quasilinear parabolic equation [6,7]. The approximate solution of the Gamma equation is the main goal of this study.

In the theory of difference schemes [8], the maximum principle is of great interest. In particular, it is used to study the stability and convergence of a difference solution on a uniform norm. Computational methods that satisfy the maximum principle are called monotone. Monotone schemes play an important role in computational practice, since the corresponding discrete problems are well-posed [9]. Moreover they provide numerical solution without oscillations even in the case of non-smooth solutions [10].

It is non-less important that one can obtain lower estimates of the solutions to differential difference problems, or in the general case, two-sided estimates for the solution of the problems. This is especially important for investigation of theoretical properties of the computational methods approximating problems with unbounded nonlinearities, where it is necessary to prove that discrete solution belongs to a neighbourhood of the exact solution. As an example we investigate the Gamma equation modelling pricing of options in financial mathematics. In this context, it is interesting to note the paper [11], in which two-sided estimates for solution of difference schemes approximating Dirichlet problem for linear parabolic equation are obtained in the discrete and continuous cases.

In the present paper, the Gamma equation is considered, on the basis of the technique from [12], two-sided estimates are obtained for its exact solution. The obtained results are generalized to the construction of unconditionally monotone finite-difference schemes of second-order of local approximation on uniform grids for a given equation. The construction of such schemes is based on the appropriate choice of the perturbed coefficient, similarly to [8]. Using the difference maximum principle, two-sided and a priori estimates are obtained in the C-norm for the difference solution. It is interesting to note that the proved two-sided estimates of the difference solution are completely consistent with the estimates of exact solution of differential problem.

\section{Auxiliary results}

Assume that $\Omega_{h}$ is a finite set of nodes (grid) in some bounded domain of the $n$-dimensional Euclidean space, and $x \in \Omega_{h}$ is a point of the grid $\Omega_{h}$. Consider the equation

$$
A(x) y(x)=\sum_{\xi \in \mathcal{M}^{\prime}(x)} B(x, \xi) y(\xi)+F(x), \quad x \in \Omega_{h},
$$


which is called the canonical form of the finite-difference scheme [8, p. 226]. Here $\mathcal{M}^{\prime}(x)=\mathcal{M}(x) \backslash x$, and $\mathcal{M}(x)$ is the grid stencil. Since any finite-difference scheme can be written as (1), monotonicity is understood as the following conditions saying that the coefficients of Eq. (1) are positive

$$
\begin{gathered}
A(x)>0, \quad B(x, \xi)>0 \quad \text { for all } \xi \in \mathcal{M}^{\prime}(x), \\
D(x)=A(x)-\sum_{\xi \in \mathcal{M}^{\prime}(x)} B(x, \xi)>0 \text { for all } \xi \in \mathcal{M}^{\prime}(x) .
\end{gathered}
$$

To obtain a two-sided estimate of the solution of a finite-difference scheme, it is most convenient to use the following lemma.

Lemma $1([13,14])$ Assume that conditions (2)-(3) that the coefficients are positive are satisfied. Then the maximum and minimum values of the solution of the finite-difference scheme (1) belong to the range of the input data

$$
\min _{x \in \Omega_{h}} \frac{F(x)}{D(x)} \leqslant y(x) \leqslant \max _{x \in \Omega_{h}} \frac{F(x)}{D(x)}, \quad x \in \Omega_{h} .
$$

Corollary 1 ([8, p. 231]) Assume that conditions of the lemma are satisfied. Then in the grid analog of the $C$-norm, the solution of finite-difference problem (1) satisfies the estimate

$$
\|y\|_{C}=\max _{x \in \Omega_{h}}|y(x)| \leqslant\left\|\frac{F}{D}\right\|_{C} .
$$

\section{Statement of the problem and two-sided estimate of the exact solution}

In a rectangle $\bar{Q}_{T}=\left\{(x, t): l_{1} \leqslant x \leqslant l_{2}, 0 \leqslant t \leqslant T\right\}$ we consider the following initial boundary value problem for a quasilinear parabolic equation, which is called the Gamma equation [7]

$$
\frac{\partial u}{\partial t}=\frac{\partial^{2} \beta(u)}{\partial x^{2}}+\frac{\partial \beta(u)}{\partial x}+c \frac{\partial u}{\partial x}, \quad u=u(x, t), \quad c=\text { const },
$$

with homogeneous boundary conditions

$$
u\left(l_{1}, t\right)=u\left(l_{2}, t\right)=0, \quad t>0,
$$

and initial conditions

$$
u(x, 0)=u_{0}(x), l_{1} \leqslant x \leqslant l_{2} .
$$

Equation (6) can be written as

$$
\frac{\partial u}{\partial t}=\frac{\partial}{\partial x}\left(k(u) \frac{\partial u}{\partial x}\right)+r(u) \frac{\partial u}{\partial x}
$$

with coefficients

$$
k(u)=\beta^{\prime}(u), \quad r(u)=k(u)+c .
$$

We assume that parabolicity condition of equation (9) on the solution [15] is satisfied

$$
0<k_{1} \leqslant k(u) \leqslant k_{2}, \quad \forall u \in \bar{D}_{u}, \quad k_{1}, k_{2}=\text { const, }
$$

where

$$
\bar{D}_{u}=\left\{u(x, t): \quad m_{1} \leqslant u(x, t) \leqslant m_{2}, \quad(x, t) \in \bar{Q}_{T}\right\} .
$$

We assume in what follows that there exists a unique solution of problem (6)-(8) and all coefficients in Eq. (9) and the desired function have continuous bounded derivatives of order that is required as the presentation proceeds.

Using the technique from [12], we prove two-sided estimates for the exact solution of problem (6)-(8). 
Theorem 1. Let condition (11) be satisfied. Then for solution $u(x, t)$ of problem (6)-(8) the following two-sided estimates are true:

$$
m_{1}=\min \left\{0, \min _{l_{1} \leqslant x \leqslant l_{2}} u_{0}(x)\right\} \leqslant u(x, t) \leqslant \max \left\{0, \max _{l_{1} \leqslant x \leqslant l_{2}} u_{0}(x)\right\}=m_{2} .
$$

Proof. To prove (12), we make the transformation of the function $u(x, t)$ to the new function $v(x, t)$ associated with it by the equality

$$
u(x, t)=v(x, t) e^{\lambda t}
$$

where $\lambda$ is an arbitrary number. The function $v(x, t)$ satisfies the equation

$$
\frac{\partial v}{\partial t}+\lambda v-k\left(v e^{\lambda t}\right) \frac{\partial^{2} v}{\partial x^{2}}-\frac{\partial k\left(v e^{\lambda t}\right)}{\partial x} \frac{\partial v}{\partial x}-r\left(v e^{\lambda t}\right) \frac{\partial v}{\partial x}=0,
$$

with initial and boundary conditions

$$
\begin{aligned}
& v(x, 0)=u_{0}(x), \quad l_{1} \leqslant x \leqslant l_{2}, \\
& v\left(l_{1}, t\right)=v\left(l_{2}, t\right)=0, \quad t>0 .
\end{aligned}
$$

Let the maximum of the solution $v(x, t)$ of problem (13)-(15) be reached at some point $\left(x_{0}, t_{0}\right) \in$ $\left(l_{1}, l_{2}\right) \times(0, T]$

$$
\max _{(x, t) \in \bar{Q}_{T}} v(x, t)=v\left(x_{0}, t_{0}\right),
$$

moreover, at the point $\left(x_{0}, t_{0}\right)$ equation (13) and the following relations are satisfied

$$
\begin{gathered}
\frac{\partial v\left(x_{0}, t_{0}\right)}{\partial t} \geqslant 0, \quad \frac{\partial v\left(x_{0}, t_{0}\right)}{\partial x}=0, \\
\frac{\partial^{2} v\left(x_{0}, t_{0}\right)}{\partial x^{2}}=\lim _{\Delta x \rightarrow 0} \frac{v\left(x_{0}-\Delta x, t_{0}\right)-2 v\left(x_{0}, t_{0}\right)+v\left(x_{0}+\Delta x, t_{0}\right)}{\Delta x^{2}} \leqslant 0 .
\end{gathered}
$$

It follows that

$$
v(x, t) \leqslant v\left(x_{0}, t_{0}\right) \leqslant 0, \quad \lambda>0 .
$$

If the maximal in $\bar{Q}_{T}$ value $v(x, t)$ is taken at the boundary $\left\{l_{1}, l_{2}\right\} \times(0, T] \cup\left[l_{1}, l_{2}\right] \times\{0\}$, then we get

$$
v(x, t) \leqslant \max _{(x, t) \in Q_{T}} v(x, t)=\max \left\{0, \max _{l_{1} \leqslant x \leqslant l_{2}} u_{0}(x)\right\} .
$$

Thus, in all cases (16)-(17) the following estimate is valid

from which it follows

$$
v(x, t) \leqslant \max \left\{0, \max _{l_{1} \leqslant x \leqslant l_{2}} u_{0}(x)\right\},
$$

$$
u(x, t) \leqslant e^{\lambda T} \max \left\{0, \max _{l_{1} \leqslant x \leqslant l_{2}} u_{0}(x)\right\}, \lambda>0 .
$$

When $\lambda \rightarrow 0$ we get the right-hand side of inequalities (12). The case of the minimum of the solution $u(x, t)$ is proved similarly. The theorem is proved.

\section{Unconditionally monotone finite-difference scheme of second order approximation on uniform grids for the Gamma equation}

Using the principle of regularization [8] on a regular uniform grid in space and time

$$
\bar{\omega}=\bar{\omega}_{h} \times \bar{\omega}_{\tau}, \quad \bar{\omega}_{h}=\left\{x_{i}=l_{1}+i h, \quad i=\overline{0, N}, \quad h N=l_{2}-l_{1}\right\}, \quad \bar{\omega}_{h}=\omega_{h} \cup\left\{x_{0}=l_{1}, x_{N}=l_{2}\right\},
$$




$$
\bar{\omega}_{\tau}=\left\{t_{n}=n \tau, \quad n=\overline{0, N_{0}}, \quad \tau N_{0}=T\right\}, \quad \bar{\omega}_{\tau}=\omega_{\tau} \cup\left\{t_{N_{0}}=T\right\},
$$

we approximate equation (9) with a difference scheme of the form

where

$$
\begin{aligned}
& \frac{y_{i}^{n+1}-y_{i}^{n}}{\tau}=\frac{\kappa_{i}^{n}(y)}{h}\left(a_{i+1}^{n}(y) \frac{y_{i+1}^{n+1}-y_{i}^{n+1}}{h}-a_{i}^{n}(y) \frac{y_{i}^{n+1}-y_{i-1}^{n+1}}{h}\right)+ \\
& +b_{i}^{+}(y) a_{i+1}^{n}(y) \frac{y_{i+1}^{n+1}-y_{i}^{n+1}}{h}+b_{i}^{-}(y) a_{i}^{n}(y) \frac{y_{i}^{n+1}-y_{i-1}^{n+1}}{h}, \\
& y_{i}^{0}=u_{0}\left(x_{i}\right), \quad y_{0}^{n+1}=y_{N}^{n+1}=0,
\end{aligned}
$$

$$
\begin{gathered}
\kappa_{i}^{n}(y)=\left(1+R_{i}^{n}(y)\right)^{-1}, \quad R_{i}^{n}(y)=\frac{0.5 h\left|r\left(y_{i}^{n}\right)\right|}{k\left(y_{i}^{n}\right)} \geqslant 0, \\
b_{i}^{+}(y)=\frac{r^{+}\left(y_{i}^{n}\right)}{k\left(y_{i}^{n}\right)} \geqslant 0, \quad b_{i}^{-}(y)=\frac{r^{-}\left(y_{i}^{n}\right)}{k\left(y_{i}^{n}\right)} \leqslant 0, \\
r^{+}\left(y_{i}^{n}\right)=0.5\left(r\left(y_{i}^{n}\right)+\left|r\left(y_{i}^{n}\right)\right|\right) \geqslant 0, \quad r^{-}\left(y_{i}^{n}\right)=0.5\left(r\left(y_{i}^{n}\right)-\left|r\left(y_{i}^{n}\right)\right|\right) \leqslant 0, \\
a_{i+1}^{n}(y)=0.5\left(k\left(y_{i+1}^{n}\right)+k\left(y_{i}^{n}\right)\right), \quad a_{i}^{n}(y)=0.5\left(k\left(y_{i-1}^{n}\right)+k\left(y_{i}^{n}\right)\right) .
\end{gathered}
$$

Approximation error. The approximation error of the difference scheme (18) has the form

$$
\psi=-u_{t}+\kappa(u)\left(a(u) \hat{u}_{\bar{x}}\right)_{x}+b^{+}(u) a^{(+1)}(u) \hat{u}_{x}+b^{-}(u) a(u) \hat{u}_{\bar{x}},
$$

where

$$
\begin{gathered}
v=v^{n}=v\left(t_{n}\right), \quad \hat{v}=v^{n+1}=v\left(t_{n+1}\right), \quad v_{x}=\frac{\left(v_{i+1}-v_{i}\right)}{h}, \\
v_{\bar{x}}=\frac{\left(v_{i}-v_{i-1}\right)}{h}, \quad a^{(+1)}(u)=a_{i+1}(u), \quad a(u)=a_{i}(u) .
\end{gathered}
$$

Taking into account

$$
\begin{gathered}
b^{+}(u)=\frac{r^{+}(u)}{k(u)}, \quad b^{-}(u)=\frac{r^{-}(u)}{k(u)}, \\
r^{+}(u)+r^{-}(u)=r(u), \quad r^{+}(u)-r^{-}(u)=|r(u)|, \\
u_{t}=\frac{\partial u}{\partial t}+O(\tau), \quad\left(a(u) \hat{u}_{\bar{x}}\right)_{x}=\frac{\partial}{\partial x}\left(k(u) \frac{\partial u}{\partial x}\right)+O\left(h^{2}+\tau\right), \\
a^{(+1)}(u) \hat{u}_{x}=k(u) \frac{\partial u}{\partial x}+0.5 h \frac{\partial}{\partial x}\left(k(u) \frac{\partial u}{\partial x}\right)+O\left(h^{2}+\tau\right), \\
a(u) \hat{u}_{\bar{x}}=k(u) \frac{\partial u}{\partial x}-0.5 h \frac{\partial}{\partial x}\left(k(u) \frac{\partial u}{\partial x}\right)+O\left(h^{2}+\tau\right),
\end{gathered}
$$

we get

$$
b^{+}(u) a^{(+1)}(u) \hat{u}_{x}+b^{-}(u) a(u) \hat{u}_{\bar{x}}=r(u) \frac{\partial u}{\partial x}+R(u) \frac{\partial}{\partial x}\left(k(u) \frac{\partial u}{\partial x}\right)+O\left(h^{2}+\tau\right) .
$$

It follows from (19) that

$$
\psi=\frac{(R(u))^{2}}{1+R(u)} \frac{\partial}{\partial x}\left(k(u) \frac{\partial u}{\partial x}\right)+O\left(h^{2}+\tau\right)=O\left(h^{2}+\tau\right) .
$$

Therefore the difference scheme (18) has second order of approximation with respect to space and first order with respect to time.

\section{Monotonicity, two-sided and a priori estimates}

We write the difference scheme (18) in the canonical form (1)

$$
A_{i}^{n} y_{i-1}^{n+1}-C_{i}^{n} y_{i}^{n+1}+B_{i}^{n} y_{i+1}^{n+1}=-F_{i}^{n}, i=1,2, \ldots, N-1,
$$


with coefficients defined as follows

$$
y_{0}^{n+1}=y_{N}^{n+1}=0
$$

$$
\begin{gathered}
A_{i}^{n}=\frac{\tau}{h^{2}} a_{i}^{n}(y)\left(\kappa_{i}^{n}(y)-h b_{i}^{-}(y)\right), \quad B_{i}^{n}=\frac{\tau}{h^{2}} a_{i+1}^{n}(y)\left(\kappa_{i}^{n}(y)+h b_{i}^{+}(y)\right), \\
C_{i}^{n}=1+A_{i}^{n}+B_{i}^{n}, \quad F_{i}^{n}=y_{i}^{n}, \quad D_{i}^{n}=C_{i}^{n}-A_{i}^{n}-B_{i}^{n}=1, \quad i=\overline{1, N-1 .}
\end{gathered}
$$

The scheme (20)-(21) is monotone if the positivity conditions of the coefficients (2)-(3) are satisfied [8], i.e. if

$$
A_{i}^{n}>0, \quad B_{i}^{n}>0, \quad D_{i}^{n}=C_{i}^{n}-A_{i}^{n}-B_{i}^{n}>0 .
$$

We need to prove that $a_{i}^{n}(y)>0$ for all $i, n$. In fact, when $n=0$, it is obvious that $a_{i}^{0}(y)=$ $0.5\left(k\left(u_{0 i}\right)+k\left(u_{0 i-1}\right)\right)>0$. Assume that, for any arbitrary $n, a_{i}^{n}(y)>0$ is also true. From this assumption we have $A_{i}^{n}>0, B_{i}^{n}>0, C_{i}^{n}>0$. According to Lemma 1 on the base of the estimate (4) for arbitrary $t=t_{n} \in \omega_{\tau}$ and all $i=0,1, \ldots, N$, we have

$$
\min \left\{0, \min _{1 \leqslant i \leqslant N-1} y_{i}^{n}\right\} \leqslant y_{i}^{n+1} \leqslant \max \left\{0, \max _{1 \leqslant i \leqslant N-1} y_{i}^{n}\right\} .
$$

Using induction on $n$, from (22) we obtain the two-sided estimate via the input data without assumption for sign-definiteness of input data

$$
\min \left\{0, \min _{l_{1} \leqslant x \leqslant l_{2}} u_{0}(x)\right\} \leqslant y_{i}^{n+1} \leqslant \max \left\{0, \max _{l_{1} \leqslant x \leqslant l_{2}} u_{0}(x)\right\}, \quad i=0,1, \ldots N .
$$

In view of (23) we obtain $y_{i}^{n+1} \in \bar{D}_{u}$, i.e. $a_{i}^{n+1}(y)=0.5\left(k\left(y_{i}^{n+1}\right)+k\left(y_{i-1}^{n+1}\right)\right)>0$. Since all positivity conditions for the coefficients (2)-(3) are satisfied, then the difference scheme (18) is monotone for all $h$ and $\tau$ (i.e. unconditionally monotone). Therefore, the following theorem is proved.

Theorem 2. Suppose that the conditions (11) are fulfilled. Then the finite-difference scheme (18) is unconditionally monotone and for its solution $y \in \bar{D}_{u}$ the above two-sided estimates (23) hold. norm

On the basis of the maximum principle in a standard way we obtain the a priori estimate in the $C$ -

Theorem 3. Let the condition (11) be fulfilled. Then for the solution of the difference problem (18) the following a priori estimate holds

$$
\left\|y^{n}\right\|_{\bar{C}} \leqslant\left\|u_{0}\right\|_{\bar{C}} .
$$

Proof. Since all the coefficients of the scheme satisfy inequalities (2)-(3), on the base of Corollary 1 we have $\left\|y^{n+1}\right\|_{\bar{C}} \leqslant\left\|y^{n}\right\|_{\bar{c}}$. Hence, we obtain the chain of relations

$$
\left\|y^{n+1}\right\|_{\bar{C}} \leqslant\left\|y^{n}\right\|_{\bar{C}} \leqslant\left\|y^{n-1}\right\|_{\bar{C}} \leqslant \cdots \leqslant\left\|u_{0}\right\|_{\bar{C}} .
$$

The theorem is proved.

Remark 1. It is interesting to note that the maximal and minimal values of the difference solution do not depend on the diffusion coefficient $k(u)$ and the convection coefficient $r(u)$.

Remark 2. For the case of $c=0$, equation (9) can be written as

$$
\frac{\partial u}{\partial t}=e^{-x} \frac{\partial}{\partial x}\left(\bar{k}(x, u) \frac{\partial u}{\partial x}\right), \quad \bar{k}(x, u)=e^{x} k(u), \quad k(u)=\beta^{\prime}(u) .
$$

Then, as construction of monotone difference schemes for it, we do not need to use the regularization principle.

Remark 3. The estimates obtained in (23) are fully consistent with the estimates of exact solution of differential problem (12). 
An example of the function $\boldsymbol{\beta}(\boldsymbol{u})$. For the case of the Frey model [7] $\beta(u)=u /(1-\rho u)^{2}, \rho>0$ from (10) we obtain the coefficient $k(u)$ of the form $k(u)=(1+\rho u) /(1-\rho u)^{3}$. Then, by virtue of (11), equation (6) will be parabolic if $k(u)>0, \forall u \in \bar{D}_{u}$, i.e. if

$$
-\frac{1}{\rho}<u(x, t)<\frac{1}{\rho}
$$

Obviously, for solution of the difference scheme (18), which approximates problem (6)-(8), conditions (24) are fulfilled, because by Theorem 1 for all $i=0,1,2, \ldots, N, n=0,1,2, \ldots, N_{0}$ we have

$$
-\frac{1}{\rho}<\min \left\{0, \min _{l_{1} \leqslant x \leqslant l_{2}} u_{0}(x)\right\} \leqslant y_{i}^{n} \leqslant \max \left\{0, \max _{l_{1} \leqslant x \leqslant l_{2}} u_{0}(x)\right\}<\frac{1}{\rho} .
$$

Numerical experiment. We consider the particular case of Gamma equation with homogeneous boundary conditions

$$
\begin{aligned}
& \frac{\partial u}{\partial t}=\frac{\partial}{\partial x}\left(\frac{1+u}{(1-u)^{3}} \frac{\partial u}{\partial x}\right), \quad \rho=1, \quad 0<x<\pi, \quad 0<t \leq 1, \\
& u(x, 0)=\sin x, \quad u(0, t)=u(\pi, t)=0 .
\end{aligned}
$$

As the coefficient $k(u)=\frac{(1+u)}{(1-u)^{3}}$ is not defined at $u=1$, then it is not defined for the initial function $u_{0}(x)=\sin x$ at $x=x^{*}=\frac{\pi}{2}$. So we build uniform grid with step $h=\frac{\pi}{(2 N+1)}$ in order to $x_{i} \neq x^{*}$. The approximate solution of the problem (25) at $t=1$, obtained by the difference scheme (18), is shown on Fig. 1.

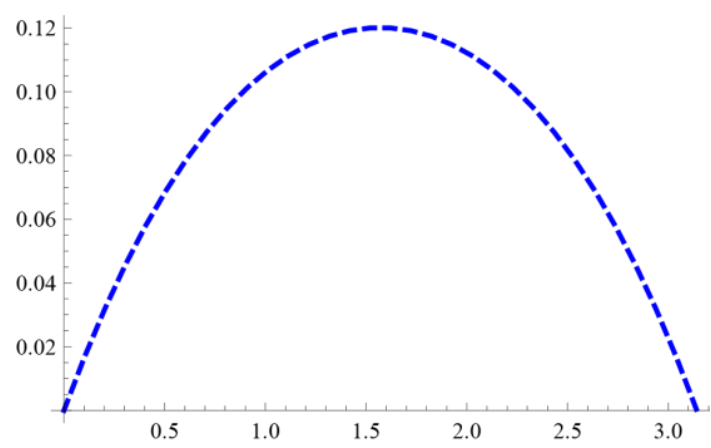

Figure 1. Numerical solution at $t=1$ with step $h=\frac{\pi}{31} \approx 0.1$ and $\tau=0.1$

Remark 4: The best numerical results are obtained if the extremal point is not a node of grid. The numerical solution is not defined, if $\mathrm{x}=\mathrm{x}^{*}$ is a node of the grid. The solution presented on Fig. 1 is not mathematically correct because the solution of (25) is not defined for such choice of the initial data. Therefore it is important to construct grid domain so that an extremal point is a grid node.

\section{Conclusions}

In this paper we develop unconditionally monotone finite-difference schemes of second-order of local approximation on uniform grids, based on regularization principle, for the initial boundary problem value for the Gamma equation. Two-side estimates of the solution of the scheme are established. Such estimates permit not only to prove the non-negativity of the exact solution, but also to find sufficient conditions on the input data when the nonlinear problem is parabolic. As a result a priori estimates of the approximate solution in the grid norm $C$ that depend on the initial and boundary conditions only are proved. 


\section{Acknowledgments}

This work was supported by University of Economics - The University of Danang (Project T2019 - 04 - 43).

\section{References}

[1] O. Hyong-Chol, J.J. Jo, J.S. Kim, General properties of solutions to inhomogeneous Black-Scholes equations with discontinuous maturity payoffs, Journal of Differential Equations 260 (4) (2016) 3151-3172. https://doi.org/10.1016/j.jde.2015.08.036.

[2] F. Black, M. Scholes, The pricing of options and corporate liabilities, Journal of political economy 81 (3) (1973) 637-654.

[3] G. Barles, H.M. Soner, Option pricing with transaction costs and a nonlinear Black-Scholes equation, Finance Stoch 2 (4) (1998) 369-397. https://doi.org/10.1007/s007800050046.

[4] R. Company, L. Jodar, E. Ponsoda, C. Ballester, Numerical analysis and simulation of option pricing problems modeling illiquid markets, Comput. Math. Appl. 59 (2010) 2964-2975. https://doi.org/10.1016/j.camwa.2010.02.014.

[5] E. Demkova, M. Ehrhardt, A high-order compact merhod for nonlinear Black-Scholes option pricing of American options, Int. J. Comput. Math. 88 (13) (2011) 2782-2797. https://doi.org/10.1080/00207160.2011.558574.

[6] M. Jandacka, D. Sevcovic, On the risk-adjusted pricing-methodology-based valuation of vanilla options and explanation of the volatility smile, J. of Appl. Math. 3 (2005) 235-258. http://dx.doi.org/10.1155/JAM.2005.235.

[7] M.N. Koleva, L.G. Vulkov, A second-order positivity preserving numerical method for Gamma equation, Appl. Math. and Comput. 220 (2013) 722-734. https://doi.org/10.1016/j.amc.2013.06.082.

[8] A. Samarskii, The Theory of Difference Schemes, Marcel Dekker Inc., New York, Basel, 2001.

[9] S. Godunov, V. Ryabenkii, Difference Schemes, Nauka, Moscow, 1977 (in Russian).

[10] P. Matus, V.T.K. Tuyen, F. Gaspar, Monotone difference schemes for linear parabolic equations with mixed boundary conditions, Dokl. Natl. Acad. Sci. Belarus 58 (5) (2014), 18-22 (in Russian).

[11] I. Farago, R. Horvath, Discrete maximum principle and adequate discretizations of linear parabolic problems, SIAM J. Sci. Comput. 28 (6) (2006), 2313-2336. https://doi.org/10.1137/050627241.

[12] O. A. Ladyzhenskaya, V. A. Solonnikov, N. N. Uraltseva, Lineinye i kvazilineinye uravneniya parabolicheskogo tipa (Linear and Quasilinear Equations of Parabolic Type), Moscow: Nauka, 1967.

[13] P. Matus, L.M. Hieu, L.G. Vulkov, Maximum principle for finite-difference schemes with non sign-constant input data, Dokl. Nats. Akad. Nauk Belarusi. 59 (5) (2015) 13-17 (in Russian).

[14] P. Matus, L.M. Hieu, L.G. Vulkov, Analysis of second order difference schemes on non-uniform grids for quasilinear parabolic equations, J. of Comput. Appl. Math. 310 (2017) 186-199. https://doi.org/10.1016/j.cam.2016.04.006.

[15] A. Friedman, Partial Differential Equations of Parabolic Type, Prentice-Hall, Englewood Cliffs, 1964. 